\title{
COMMON FIXED POINT THEOREM IN INTUITIONISTIC FUZZY METRIC SPACE USING COMPATIBLE MAPPINGS OF TYPE (K)
}

\author{
${ }^{1} \mathrm{~K}$. B. Manandhar, ${ }^{1} \mathrm{~K}$. Jha and ${ }^{2} \mathrm{Y}$.J. Cho \\ ${ }^{1}$ Department of Natural Sciences (Mathematics), School of Science \\ Kathmandu University, Dhulikhel, NEPAL \\ ${ }^{2}$ Department of Mathematics, Gyeongsang National University \\ Chinju, South Korea
}

Keywords: Intuitionistic fuzzy metric space, weakly compatible, compatible mappings of type (K)

\begin{abstract}
In this paper, we introduce the notion of compatible mappings of type $(\mathrm{K})$ in intuitionistic fuzzy metric space and obtain a common fixed point theorem for self mappings on complete intuitionistic fuzzy metric space with example. Our result generalizes and improves other similar results in literature.
\end{abstract}

\section{Introduction}

Fixed point theory is an important area of functional analysis. The study of common fixed point of mappings satisfying contractive type conditions has been a very active field of research. In 1965, the concept of fuzzy set was introduced by L.A Zade[11]. Then, fuzzy metric spaces have been introduced by Kramosil and Michalek [13]. George and Veeramani [1] modified the notion of fuzzy metric spaces with the help of continuous t-norms. In 1986, G. Jungck [5] introduced notion of compatible mappings. In 1993, G. Jungck, P. P. Murthy and Y. J. Cho [6] gave a generalization of compatible mappings called compatible mappings of type (A) which is equivalent to the concept of compatible mappings under some conditions. In 1996, H. K. Pathak, Y. J. Cho, S. S. Chang and S. M. Kang [7] introduced the concept of compatible mappings of type (P) and compared with compatible mappings of type (A) and compatible mappings. In 1998, R. P. Pant [14] introduced the notion of reciprocal continuity of mappings in metric spaces. In 1998, Y.J. Cho, H.K. Pathak, S.M. Kang and J.S. Jung [16] introduced the concept of compatible mappings in fuzzy metric space.

Recently, Jha et al. [10] introduced the concept of compatible mappings of type (K) in metric space and shows that the compatible mapping of type $(\mathrm{K})$ is independent with other compatible. Also, Manandhar et al. [9] introduced compatible mapping of type $(\mathrm{K})$ in fuzzy metric space. Many authors have obtained fixed point theorems in fuzzy metric space using these compatible notions.

The purpose of this paper is to establish a common fixed point theorem for compatible mappings of type $(\mathrm{K})$ in intuitionistic fuzzy metric spaces with example.

\section{Preliminaries}

Definition 2.1. [12] Let $X$ be any non empty set. A fuzzy set $A$ in $X$ is a function with domain $X$ and values in $[0,1]$.

Definition 2.2. [1] A binary operation $*:[0,1] \times[0,1] \rightarrow[0,1]$ is a continuous t-norm if $*$ is satisfying the following conditions:

(a) $*$ is commutative and associative;

(b) $*$ is continuous;

(c) $a * 1=a$ for all a $\in[0,1]$;

(d) $a * b \leq c * d$ whenever $a \leq c$ and $b \leq d$, and $a, b, c, d \in[0,1]$.

Definition 2.3. [2] A binary operation $\diamond:[0,1] \times[0,1] \rightarrow[0,1]$ is a continuous t-conorm if it satisfies the following conditions:

(a) $\diamond$ is commutative and associative; 
(b) $\diamond$ is continuous;

(c) a $\diamond 0=$ a for all $\mathrm{a} \in[0,1]$;

(d) $\mathrm{a} \diamond \mathrm{b} \leq \mathrm{c} \diamond \mathrm{d}$ whenever $\mathrm{a} \leq \mathrm{c}$ and $\mathrm{b} \leq \mathrm{d}$, for each $\mathrm{a}, \mathrm{b}, \mathrm{c}, \mathrm{d} \in[0,1]$.

Definition 2.4. [3] A 5-tuple (X, M, N, *, $\diamond)$ is said to be an intuitionistic fuzzy metric space (shortly IFM-Space) if $\mathrm{X}$ is an arbitrary set, $*$ is a continuous t-norm $\diamond$ is a continuous $\mathrm{t}$-conorm and $\mathrm{M}, \mathrm{N}$ are fuzzy sets on $\mathrm{X}^{2} \times(0, \infty)$ satisfying the following conditions: for all $\mathrm{x}, \mathrm{y}, \mathrm{z} \in \mathrm{X}$ and $\mathrm{s}, \mathrm{t}>0$;

$($ IFM-1) $M(x, y, t)+N(x, y, t) \leq 1$;

$(\mathrm{IFM}-2) \mathrm{M}(\mathrm{x}, \mathrm{y}, 0)=0$

$($ IFM-3) $M(x, y, t)=1$ if and only if $x=y$;

(IFM-4) $M(x, y, t)=M(y, x, t)$;

$($ IFM-5) M(x, y, t) * M (y, z, s) $\leq M(x, z, t+s)$

(IFM-6) $\mathrm{M}(\mathrm{x}, \mathrm{y},):.[0, \infty) \rightarrow[0,1]$ is left continuous;

$\left(\right.$ IFM-7) $\lim _{\mathrm{t} \rightarrow \infty} \mathrm{M}(\mathrm{x}, \mathrm{y}, \mathrm{t})=1$

$($ IFM-8) N(x, y, 0) = 1;

(IFM-9) $N(x, y, t)=0$ if and only if $x=y$;

$($ IFM-10) $N(x, y, t)=N(y, x, t)$;

$($ IFM-11) N(x, y, t $) \diamond \mathrm{N}(\mathrm{y}, \mathrm{z}, \mathrm{s}) \geq \mathrm{N}(\mathrm{x}, \mathrm{z}, \mathrm{t}+\mathrm{s})$;

$($ IFM-12) $\mathrm{N}(\mathrm{x}, \mathrm{y},):.[0, \infty) \rightarrow[0,1]$ is right continuous;

$\left(\right.$ IFM-13) $\lim _{\mathrm{t} \rightarrow \infty} \mathrm{N}(\mathrm{x}, \mathrm{y}, \mathrm{t})=0$

Then $(M, N)$ is called an intuitionistic fuzzy metric on $X$. The functions $M(x, y, t)$ and $N(x, y, t)$ denote the degree of nearness and degree of non-nearness between $\mathrm{x}$ and $\mathrm{y}$ with respect to $\mathrm{t}$, respectively.

Remark 2.5. [12] Every fuzzy metric space (X, M, *) is an intuitionistic fuzzy metric space if $X$ of the form $(\mathrm{X}, \mathrm{M}, 1-\mathrm{M}, *, \diamond)$ such that $\mathrm{t}$ - norm * and t-conorm $\diamond$ are associated, that is, $\mathrm{x} \diamond \mathrm{y}=1-((1-$ $\mathrm{x}) *(1-\mathrm{y}))$ for any $\mathrm{x}, \mathrm{y} \in \mathrm{X}$. But the converse is not true.

Example 2.6. [14] Let $(X, d)$ be a metric space. Denote $a * b=a b$ and $a \diamond b=\min \{1, a+b\}$ for all $a, b \in[0,1]$ and let $M_{d}$ and $N_{d}$ be fuzzy sets on $X^{2} \times(0, \infty)$ defined as follows;

$M_{d}(x, y, t)=\frac{t}{t+d(x, y)} ; N_{d}(x, y, t)=\frac{d(x, y)}{t+d(x, y)}$.

Then $\left(\mathrm{M}_{\mathrm{d}}, \mathrm{N}_{\mathrm{d}}\right)$ is an intuitionistic fuzzy metric on $\mathrm{X}$. We call this intuitionistic fuzzy metric induced by a metric $\mathrm{d}$ the standard intuitionistic fuzzy metric.

Remark 2.7. Note the above example holds even with the t-norm $a * b=\min \{a, b\}$ and the $t$ conorm $\mathrm{a} \diamond \mathrm{b}=\max \{\mathrm{a}, \mathrm{b}\}$ and hence $\left(\mathrm{M}_{\mathrm{d}}, \mathrm{N}_{\mathrm{d}}\right)$ is an intuitionistic fuzzy metric with respect to any continuous t-norm and continuous t- conorm.

Definition 2.8. [3] Let $(X, M, N, *, \diamond)$ be an intuitionistic fuzzy metric space.

(a) A sequence $\{x n\}$ in $X$ is called cauchy sequence if for each $t>0$ and $P>0, \lim _{n \rightarrow \infty} M\left(x_{n+p}\right.$, $\left.x_{n}, t\right)=1$ and $\lim _{n \rightarrow \infty} N\left(x_{n+p}, x_{n}, t\right)=0$.

(b)A sequence $\left\{x_{n}\right\}$ in $X$ is convergent to $x \in X$ if $\lim _{n \rightarrow \infty} M\left(x_{n}, x, t\right)=1$ and $\lim _{n \rightarrow \infty} N\left(x_{n}, x, t\right)=$ 0 for each $t>0$.

(c) An intuitionistic fuzzy metric space is said to be complete if every Cauchy sequence is convergent.

Definition 2.9 [4] The self mappings $A$ and $S$ is called weakly compatible mapping if they commutative in their coincident point.

Definition 1.10.[10] The self mappings $A$ and $S$ of a fuzzy metric space $(X, M, *)$ are said to be compatible of type (E) iff $\lim _{n \rightarrow \infty} \mathrm{M}\left(\mathrm{AAx}_{\mathrm{n}}, \mathrm{ASx}_{\mathrm{n}}, \mathrm{t}\right)=1, \lim _{\mathrm{n} \rightarrow \infty} \mathrm{M}\left(\mathrm{AAx}_{\mathrm{n}}, \mathrm{Sx}, \mathrm{t}\right)=1$, 
$\lim _{n \rightarrow \infty} M\left(A S x_{n}, S x, t\right)=1$ and $\lim _{n \rightarrow \infty} M\left(S S x_{n}, S A x_{n}, t\right)=1, \lim _{n \rightarrow \infty} M\left(S_{n} x_{n}, A x, t\right)=1$, $\lim _{\mathrm{n} \rightarrow \infty} \mathrm{M}\left(\mathrm{SSx}_{\mathrm{n}}, \mathrm{Ax}, \mathrm{t}\right)=1$.

Definition 2.11.[10] The self mappings $A$ and $S$ of a metric space $(X, d)$ are said to be compatible of type (K) iff $\lim _{\mathrm{n} \rightarrow \infty} \mathrm{AA} x_{\mathrm{n}}=\mathrm{S} x$ and $\lim _{\mathrm{n} \rightarrow \infty} \mathrm{SS} x_{\mathrm{n}}=\mathrm{A} x$, whenever $\{x \mathrm{n}\}$ is a sequence in $\mathrm{X}$ such that $\lim _{\mathrm{n} \rightarrow \infty} \mathrm{A} x_{\mathrm{n}}=\lim _{\mathrm{n} \rightarrow \infty} \mathrm{S} x_{\mathrm{n}}=x$ for some $x$ in $\mathrm{X}$.

Definition 2.12 The self mappings $A$ and $S$ of a intuitionistic fuzzy metric space $(X, M, N, *, \diamond)$ are said to be compatible of type (K) iff $\lim _{\mathrm{n} \rightarrow \infty} \mathrm{M}\left(\mathrm{AA} x_{\mathrm{n}}, \mathrm{S} x, \mathrm{t}\right)=1$ and $\lim _{\mathrm{n} \rightarrow \infty} \mathrm{M}\left(\mathrm{SS} x_{\mathrm{n}}, \mathrm{A} x, \mathrm{t}\right)=1$, whenever $\{x \mathrm{n}\}$ is a sequence in $\mathrm{X}$ such that $\lim _{\mathrm{n} \rightarrow \infty} \mathrm{A} x_{\mathrm{n}}=\lim _{\mathrm{n} \rightarrow \infty} \mathrm{S} x_{\mathrm{n}}=x$ for some $x$ in $\mathrm{X}$ and $\mathrm{t}>0$.

Lemma 2.13. [8] In an intuitionistic fuzzy metric space $X, M(x, y,$.$) is non-decreasing and N(x, y,$. is non increasing for all $\mathrm{x}, \mathrm{y} \in \mathrm{X}$.

Lemma 2.14. [16] Let $(X, M, N, *, \diamond)$ be an intuitionistic fuzzy metric space. If there exists a constant $k \in(0,1)$ such that, $M\left(y_{n+2}, y_{n+1}, k t\right) \geq M\left(y_{n+1}, y n, t\right), N\left(y_{n+2}, y n+1, k t\right) \leq N\left(y_{n+1}, y n, t\right)$ for every $\mathrm{t}>0$ and $\mathrm{n}=1,2, \ldots$ then $\left\{\mathrm{y}_{\mathrm{n}}\right\}$ is a Cauchy sequence in $\mathrm{X}$.

Lemma 2.15. [16] Let $(X, M, N, *, \diamond)$ be an intuitionistic fuzzy metric space. If there exists a constant $\mathrm{k} \in(0,1)$ such that

$M(x, y, k t) \geq M(x, y, t), N(x, y, k t) \leq N(x, y, t)$, for all $x, y \in X$. Then $x=y$.

Proposition 2.16. If $\mathrm{A}$ and $\mathrm{S}$ be compatible mappings of type $(\mathrm{K})$ on a intuitionistic fuzzy metric space $(X, M, N, *, \diamond)$ and if one of function is continuous .Then, we have

(a) $\mathrm{A}(\mathrm{x})=\mathrm{S}(\mathrm{x})$ where $\lim _{\mathrm{n} \rightarrow \infty} \mathrm{A} x_{\mathrm{n}}=\mathrm{x} \lim _{\mathrm{n} \rightarrow \infty} \mathrm{S} x_{\mathrm{n}}=\mathrm{x}$, for some point $\mathrm{x} \in \mathrm{X}$, and sequence $\{\mathrm{xn}\}$,

(b) If these exist $u \in X$ such that $\mathrm{Au}=\mathrm{Su}=\mathrm{x}$ then, $\mathrm{ASu}=\mathrm{SAu}$.

Proof : Let $\{\mathrm{xn}\}$ be a sequence of $X$ such that $\lim _{\mathrm{n} \rightarrow \infty} \mathrm{A} x_{\mathrm{n}}=\lim _{\mathrm{n} \rightarrow \infty} \mathrm{S} x_{\mathrm{n}}=\mathrm{x}$ for some $\mathrm{x}$ in $\mathrm{X}$. Then by definition of compatible of type $(\mathrm{K})$, we have $\lim _{\mathrm{n} \rightarrow \infty} \mathrm{AA} x_{\mathrm{n}}=\mathrm{S}(\mathrm{x})$. If $\mathrm{A}$ is a continuous mapping, then we get $\lim _{\mathrm{n} \rightarrow \infty} \mathrm{AA} x_{\mathrm{n}}=\mathrm{A}\left(\lim _{\mathrm{n} \rightarrow \infty} \mathrm{A} x_{\mathrm{n}}\right)=\mathrm{A}(\mathrm{x})$. This implies $\mathrm{A}(\mathrm{x})=\mathrm{S}(\mathrm{x})$. Similarly, if $\mathrm{S}$ is continuous then, we get the same result. This is the proof of part (a).

Again, suppose $\mathrm{Au}=\mathrm{Su}=\mathrm{x}$ for some $\mathrm{u} \in \mathrm{X}$. Then, $\mathrm{ASu}=\mathrm{A}(\mathrm{Su})=\mathrm{Ax}$ and

$\mathrm{SAu}=\mathrm{S}(\mathrm{Au})=\mathrm{Sx}$. From (a), we have $\mathrm{Ax}=\mathrm{Sx}$. Hence, we get $\mathrm{ASu}=\mathrm{SAu}$.

This is the proof of part (b).

\section{Main Results}

Theorem 3. 1. If $A, B, S$ and $T$ are self mapping on a complete Intuitionistic fuzzy metric space (X, $\mathrm{M}, \mathrm{N}, *, \diamond)$, satisfying the conditions:

(i) $\mathrm{A}(\mathrm{X}) \subseteq \mathrm{T}(\mathrm{X}), \mathrm{B}(\mathrm{X}) \subseteq \mathrm{S}(\mathrm{X})$.

(ii) There exists $\mathrm{k} \in(0,1)$ such that for every $\mathrm{x}, \mathrm{y} \in \mathrm{X}$ and $\mathrm{t}>0$, $M(A x, B y, k t) \geq \min \{M(S x, T y, t), M(A x, S x, t), M(B y, T y, t), M(A x, T y, t)$, $\mathrm{M}(\mathrm{Ax}, \mathrm{By}, \mathrm{t}), \mathrm{M}(\mathrm{Sx}, \mathrm{By}, \mathrm{t})\}$, and

$N(A x, B y, k t) \leq \max \{N(S x, T y, t), N(A x, S x, t), N(B y, T y, t), N(A x, T y, t)$, $\mathrm{N}(\mathrm{Ax}, \mathrm{By}, \mathrm{t}), \mathrm{N}(\mathrm{S} x, \mathrm{By}, \mathrm{t})\}$.

(iii) $\mathrm{B}$ and $\mathrm{T}$ weakly compatible mappings.

If the pair of mappings $(A, S)$ is compatible of type $(K)$ and one of the mapping is continuous then $\mathrm{A}, \mathrm{B}, \mathrm{S}$ and $\mathrm{T}$ have a unique common fixed point in $\mathrm{X}$.

Proof: let $\mathrm{x}_{0}$ be any arbitrary point in $\mathrm{X}$, construct a sequence $\left\{\mathrm{y}_{\mathrm{n}}\right\}$ in $\mathrm{X}$ such that $\mathrm{y}_{2 \mathrm{n}-1}=\mathrm{T} \mathrm{x}_{2 \mathrm{n}-1}=$ $\mathrm{Ax}_{2 \mathrm{n}-2}$ and $\mathrm{y}_{2 \mathrm{n}}=\mathrm{Sx}_{2 \mathrm{n}}=\mathrm{Bx}_{2 \mathrm{n}+1}, \mathrm{n}=1,2,3 \ldots$ This can be done by (i). By using contractive condition, we obtain 
$\mathrm{M}\left(\mathrm{y}_{2 \mathrm{n}+1}, \mathrm{y}_{2 \mathrm{n}+2}, \mathrm{kt}\right)=\mathrm{M}\left(\mathrm{A} \mathrm{x}_{2 \mathrm{n}}, \mathrm{Bx}_{2 \mathrm{n}+1}, \mathrm{kt}\right)$

$\geq \min \left\{\mathrm{M}\left(\mathrm{Sx}_{2 \mathrm{n}}, \mathrm{T} x_{2 \mathrm{n}+1}, \mathrm{t}\right), \mathrm{M}\left(\mathrm{Ax}_{2 \mathrm{n}}, \mathrm{Sx}_{2 \mathrm{n}}, \mathrm{t}\right), \mathrm{M}\left(\mathrm{Bx}_{2 \mathrm{n}+1}, \mathrm{~T} \mathrm{x}_{2 \mathrm{n}+1}, \mathrm{t}\right)\right.$,

$\left.\mathrm{M}\left(\mathrm{Ax}_{2 \mathrm{n}}, \mathrm{Tx}_{2 \mathrm{n}+1}, \mathrm{t}\right), \mathrm{M}\left(\mathrm{Ax}_{2 \mathrm{n}}, \mathrm{Bx}_{2 \mathrm{n}+1}, \mathrm{t}\right), \mathrm{M}\left(\mathrm{Sx}_{2 \mathrm{n}}, \mathrm{Bx} \mathrm{x}_{2 \mathrm{n}+1}, \mathrm{t}\right)\right\}$

$=\min \left\{M\left(y_{2 n}, y_{2 n+1}, t\right), M\left(y_{2 n+1}, y_{2 n}, t\right), M\left(y_{2 n}, y_{2 n+1}, t\right)\right.$,

$\left.M\left(y_{2 n+1}, y_{2 n+1}, t\right), M\left(y_{2 n+1}, y_{2 n}, t\right), M\left(y_{2 n}, y_{2 n}, t\right)\right\}$

$=\min \left\{M\left(y_{2 n}, y_{2 n+1}, t\right), M\left(y_{2 n+1}, y_{2 n}, t\right), M\left(y_{2 n}, y_{2 n+1}, t\right), 1, M\left(y_{2 n+1}, y_{2 n}, t\right), 1\right\}$

$=M\left(y_{2 n}, y_{2 n+1}, t\right)$, That is

$M\left(y_{2 n+1}, y_{2 n+2}, k t\right) \geq M\left(y_{2 n}, y_{2 n+1}, t\right)$,

Similarly, we have $M\left(y_{2 n}, y_{2 n+1}, k t\right) \geq M\left(y_{2 n-1}, y_{2 n}, t\right)$,

So, we get $\mathrm{M}\left(\mathrm{y}_{\mathrm{n}+2}, \mathrm{y}_{\mathrm{n}+1}, \mathrm{kt}\right) \geq \mathrm{M}\left(\mathrm{y}_{\mathrm{n}+1}, \mathrm{y}_{\mathrm{n}}, \mathrm{t}\right)$,

Also, we get

$\mathrm{N}\left(\mathrm{y}_{2 \mathrm{n}+1}, \mathrm{y}_{2 \mathrm{n}+2}, \mathrm{kt}\right)=\mathrm{N}\left(\mathrm{Ax}_{2 \mathrm{n}}, \mathrm{Bx}_{2 \mathrm{n}+1}, \mathrm{kt}\right)$

$\leq \max \left\{\mathrm{N}\left(\mathrm{Sx}_{2 \mathrm{n}}, \mathrm{T} \mathrm{x} 2_{\mathrm{n}+1}, \mathrm{t}\right), \mathrm{N}\left(\mathrm{Ax}_{2 \mathrm{n}}, \mathrm{Sx}_{2 \mathrm{n}}, \mathrm{t}\right), \mathrm{N}\left(\mathrm{Bx}_{2 \mathrm{n}+1}, \mathrm{Tx}_{2 \mathrm{n}+1}, \mathrm{t}\right)\right.$,

$\left.\mathrm{N}\left(\mathrm{A} \mathrm{x} \mathrm{x}_{2 \mathrm{n}}, \mathrm{T} \mathrm{x}_{2 \mathrm{n}+1}, \mathrm{t}\right), \mathrm{N}\left(\mathrm{Ax}_{2 \mathrm{n}}, \mathrm{Bx}_{2 \mathrm{n}+1}, \mathrm{t}\right), \mathrm{N}\left(\mathrm{Sx}_{2 \mathrm{n}}, \mathrm{Bx}_{2 \mathrm{n}+1}, \mathrm{t}\right)\right\}$

$=\max \left\{N\left(y_{2 n}, y_{2 n+1}, t\right), N\left(y_{2 n+1}, y_{2 n}, t\right), N\left(y_{2 n}, y_{2 n+1}, t\right), N\left(y_{2 n+1}, y_{2 n+1}, t\right)\right.$,

$\left.\mathrm{N}\left(\mathrm{y}_{2 \mathrm{n}+1}, \mathrm{y}_{2 \mathrm{n}}, \mathrm{t}\right), \mathrm{N}\left(\mathrm{y}_{2 \mathrm{n}}, \mathrm{y}_{2 \mathrm{n}}, \mathrm{t}\right)\right\}$

$=\max \left\{\mathrm{N}\left(\mathrm{y}_{2 \mathrm{n}}, \mathrm{y}_{2 \mathrm{n}+1}, \mathrm{t}\right), \mathrm{N}\left(\mathrm{y}_{2 \mathrm{n}+1}, \mathrm{y}_{2 \mathrm{n}}, \mathrm{t}\right), \mathrm{N}\left(\mathrm{y}_{2 \mathrm{n}}, \mathrm{y}_{2 \mathrm{n}} 1, \mathrm{t}\right), 0, \mathrm{~N}\left(\mathrm{y}_{2 \mathrm{n}+1}, \mathrm{y}_{2 \mathrm{n}}, \mathrm{t}\right), 0\right\}$

$=\mathrm{N}\left(\mathrm{y}_{2 \mathrm{n}}, \mathrm{y}_{2 \mathrm{n}+1}, \mathrm{t}\right)$, so,

$\mathrm{N}\left(\mathrm{y}_{2 \mathrm{n}+1}, \mathrm{y}_{2 \mathrm{n}+2}, \mathrm{kt}\right) \leq \mathrm{N}\left(\mathrm{y}_{2 \mathrm{n}}, \mathrm{y}_{2 \mathrm{n}+1}, \mathrm{t}\right)$.

Similarly, we have $\mathrm{N}\left(\mathrm{y}_{2 \mathrm{n}}, \mathrm{y}_{2 \mathrm{n}+1}, \mathrm{kt}\right) \leq \mathrm{N}\left(\mathrm{y}_{2 \mathrm{n}-1}, \mathrm{y}_{2 \mathrm{n}}, \mathrm{t}\right)$,

So, we get $\mathrm{N}\left(\mathrm{y}_{\mathrm{n}+2}, \mathrm{y}_{\mathrm{n}+1}, \mathrm{kt}\right) \leq \mathrm{N}\left(\mathrm{y}_{\mathrm{n}+1}, \mathrm{yn}, \mathrm{t}\right)$.

From (1), (2) and Lemma (2.13), we get that $\{\mathrm{yn}\}$ is a Cauchy sequence in $X$.

But $(\mathrm{X}, \mathrm{M}, \mathrm{N}, *, \diamond)$ is complete so there exists a point $\mathrm{z}$ in $\mathrm{X}$ such that $\left\{\mathrm{y}_{\mathrm{n}}\right\} \rightarrow \mathrm{z}$.

Also, we have $\left\{\mathrm{Ax}_{2 \mathrm{n}-2}\right\},\left\{\mathrm{T} \mathrm{x}_{2 \mathrm{n}-1}\right\},\left\{\mathrm{Sx}_{2 \mathrm{n}}\right\},\left\{\mathrm{Bx}_{2 \mathrm{n}+1}\right\} \rightarrow \mathrm{z}$.

Since, $(A, S)$ is compatible of type $(\mathrm{K})$ and one of the mapping is continuous. So, using proposition

(2.15), we get $\mathrm{Az}=\mathrm{Sz}$.

Since $\mathrm{A}(\mathrm{X}) \subseteq \mathrm{T}(\mathrm{X})$ then there exists a point $\mathrm{u}$ in $\mathrm{X}$ such that $\mathrm{Az}=\mathrm{Tu}$. Now, by contractive condition, we get,

$M(A z, B u, k t) \geq \min \{M(S z, T u, t), M(A z, S z, t), M(B u, T u, t), M(A z, T u, t), M(A z, B u, t)$, $\mathrm{M}(\mathrm{Sz}, \mathrm{Bu}, \mathrm{t})\}$

$=\min \{M(A z, A z, t), M(A z, A z, t), M(B u, A z, t), M(A z, A z, t), M(A z, A u, t)$, $\mathrm{M}(\mathrm{Az}, \mathrm{Bu}, \mathrm{t})\}$

$\mathrm{M}(\mathrm{Az}, \mathrm{Bu}, \mathrm{kt})\} \geq \mathrm{M}(\mathrm{Az}, \mathrm{Bu}, \mathrm{t})$.

Also, we have

$\mathrm{N}(\mathrm{Az}, \mathrm{Bu}, \mathrm{kt}) \leq \max \{\mathrm{N}(\mathrm{Sz}, \mathrm{Tu}, \mathrm{t}), \mathrm{N}(\mathrm{Az}, \mathrm{Sz}, \mathrm{t}), \mathrm{N}(\mathrm{Bu}, \mathrm{Tu}, \mathrm{t}), \mathrm{N}(\mathrm{A} z, \mathrm{Tu}, \mathrm{t}), \mathrm{N}(\mathrm{Az}, \mathrm{Bu}, \mathrm{t})$, $\mathrm{N}(\mathrm{Sz}, \mathrm{Bu}, \mathrm{t}\}$

$=\max \{N(A z, A z, t), N(A z, A z, t), N(B u, A z, t), N(A z, A z, t), N(A z, A u, t)$,

$\mathrm{N}(\mathrm{Az}, \mathrm{Bu}, \mathrm{t})\}$

$\mathrm{N}(\mathrm{A} \mathrm{z}, \mathrm{Bu}, \mathrm{kt}) \leq \mathrm{N}(\mathrm{Az}, \mathrm{Bu}, \mathrm{t})$

Now, from (4), (5) and Lemma (2.14), we get $\mathrm{Az}=\mathrm{Bu}$.

Thus, we get $\mathrm{Az}=\mathrm{Sz}=\mathrm{Bu}=\mathrm{Tu}$.

To prove $\mathrm{Pz}=\mathrm{z}$, we have

$M\left(A z, B x_{2 n+1}, k t\right) \geq \min \left\{M\left(S z, T x_{2 n+1}, t\right), M(A z, S z, t), M\left(B x_{2 n+1}, T x_{2 n+1}, t\right)\right.$,

$\left.\mathrm{M}\left(\mathrm{Az}, \mathrm{Tx}_{2 \mathrm{n}+1}, \mathrm{t}\right), \mathrm{M}\left(\mathrm{Az}, \mathrm{Bx}_{2 \mathrm{n}+1}, \mathrm{t}\right), \mathrm{M}\left(\mathrm{Sz}, \mathrm{Bx}_{2 \mathrm{n}+1}, \mathrm{t}\right)\right\}$.

Taking limit as $n \rightarrow \infty$, we get

$M(A z, z, k t) \geq \min \{M(S z, z, t), M(A z, S z, t), M(z, z, t), M(A z, z, t), M(A z, z, t), M(S z, z, t)\}$

$=\min \{\mathrm{M}(\mathrm{Az}, \mathrm{z}, \mathrm{t}), 1,1, \mathrm{M}(\mathrm{Az}, \mathrm{z}, \mathrm{t}), \mathrm{M}(\mathrm{Az}, \mathrm{z}, \mathrm{t}), \mathrm{M}(\mathrm{Az}, \mathrm{z}, \mathrm{t})\}$

$M(A z, z, k t) \geq M(A z, z, t)$.

$\mathrm{N}\left(\mathrm{Az}, \mathrm{Bx}_{2 \mathrm{n}+1}, \mathrm{kt}\right) \leq \max \left\{\mathrm{N}\left(\mathrm{Sz}, \mathrm{T} \mathrm{x}_{2 \mathrm{n}+1}, \mathrm{t}\right), \mathrm{N}(\mathrm{Az}, \mathrm{Sz}, \mathrm{t}), \mathrm{N}\left(\mathrm{Bx}_{2 \mathrm{n}+1}, \mathrm{~T} \mathrm{x}_{2 \mathrm{n}+1}, \mathrm{t}\right)\right.$,

$\left.\mathrm{N}\left(\mathrm{Az}, \mathrm{Tx}_{2 \mathrm{n}+1}, \mathrm{t}\right), \mathrm{N}\left(\mathrm{Az}, \mathrm{Bx}_{2 \mathrm{n}+1}, \mathrm{t}\right), \mathrm{N}\left(\mathrm{Sz}, \mathrm{Bx}_{2 \mathrm{n}+1}, \mathrm{t}\right)\right\}$

Taking limit as $n \rightarrow \infty$, we get 
$\mathrm{N}(\mathrm{Az}, \mathrm{z}, \mathrm{kt}) \leq \max \{\mathrm{N}(\mathrm{Sz}, \mathrm{z}, \mathrm{t}), \mathrm{N}(\mathrm{Az}, \mathrm{Sz}, \mathrm{t}), \mathrm{N}(\mathrm{z}, \mathrm{z}, \mathrm{t}), \mathrm{N}(\mathrm{Az}, \mathrm{z}, \mathrm{t}), \mathrm{N}(\mathrm{Az}, \mathrm{z}, \mathrm{t}), \mathrm{N}(\mathrm{Sz}, \mathrm{z}, \mathrm{t})\}$ $=\max \{\mathrm{N}(\mathrm{Az}, \mathrm{z}, \mathrm{t}), 0,0, \mathrm{~N}(\mathrm{Az}, \mathrm{z}, \mathrm{t}), \mathrm{N}(\mathrm{Az}, \mathrm{z}, \mathrm{t}), \mathrm{N}(\mathrm{Az}, \mathrm{z}, \mathrm{t})\}$

$\mathrm{N}(\mathrm{Az}, \mathrm{z}, \mathrm{kt}) \leq \mathrm{N}(\mathrm{Az}, \mathrm{z}, \mathrm{t})$.

Now, from (7), (8) and Lemma (2.14), we get, $A z=z$.

Hence, we have $\mathrm{Az}=\mathrm{Sz}=\mathrm{z}$.

So, $\mathrm{z}$ is a common fixed point of $\mathrm{A}$ and $\mathrm{S}$.

Also, we get $\mathrm{Bu}=\mathrm{Tu}=\mathrm{z}$.

Since $\mathrm{B}$ and $\mathrm{T}$ are weakly compatible, we have $\mathrm{TBu}=\mathrm{BTu}$. So, from (6), we get

$\mathrm{Tz}=\mathrm{Bz}$.

Again, we get

$M\left(A_{2 n-2}, B z, k t\right) \geq \min \left\{M\left(S x_{2 n-2}, T z, t\right), M\left(A x_{2 n-2}, S x_{2 n-2}, t\right), M(B z, T z, t)\right.$, $\left.\mathrm{M}\left(\mathrm{A} \mathrm{x}_{2 \mathrm{n}-2}, \mathrm{Tz}, \mathrm{t}\right) \mathrm{M}\left(\mathrm{A} \mathrm{x}_{2 \mathrm{n}-2}, \mathrm{Bz}, \mathrm{t}\right), \mathrm{M}\left(\mathrm{S} \mathrm{x}_{2 \mathrm{n}-2}, \mathrm{Bz}, \mathrm{t}\right)\right\}$.

Taking limit as $\mathrm{n} \rightarrow \infty$, we have

$\mathrm{M}(\mathrm{z}, \mathrm{Bz}, \mathrm{kt}) \geq \min \mathrm{M}(\mathrm{z}, \mathrm{Tz}, \mathrm{t}), \mathrm{M}(\mathrm{z}, \mathrm{z}, \mathrm{t}), \mathrm{M}(\mathrm{Bz}, \mathrm{Tz}, \mathrm{t}), \mathrm{M}(\mathrm{z}, \mathrm{Tz}, \mathrm{t}) \mathrm{M}(\mathrm{z}, \mathrm{Bz}, \mathrm{t}), \mathrm{M}(\mathrm{z}, \mathrm{Bz}, \mathrm{t})\}$

$\mathrm{M}(\mathrm{z}, \mathrm{Bz}, \mathrm{kt}) \geq \mathrm{M}(\mathrm{z}, \mathrm{Bz}, \mathrm{t})$.

$$
=\min \{\mathrm{M}(\mathrm{z}, \mathrm{Bz}, \mathrm{t}), 1,1, \mathrm{M}(\mathrm{z}, \mathrm{Bz}, \mathrm{t}) \mathrm{M}(\mathrm{z}, \mathrm{Bz}, \mathrm{t}), \mathrm{M}(\mathrm{z}, \mathrm{Bz}, \mathrm{t})\} \text {, }
$$

Also, we have

$\mathrm{N}\left(\mathrm{Ax}_{2 \mathrm{n}-2}, \mathrm{Bz}, \mathrm{kt}\right) \leq \max \left\{\mathrm{N}\left(\mathrm{S} \mathrm{x}_{2 \mathrm{n}-2}, \mathrm{~T} \mathrm{z}, \mathrm{t}\right), \mathrm{N}\left(\mathrm{A} \mathrm{x} \mathrm{x}_{2 \mathrm{n}-2}, \mathrm{~S} \mathrm{x}_{2 \mathrm{n}-2}, \mathrm{t}\right), \mathrm{N}(\mathrm{Bz}, \mathrm{T} \mathrm{z}, \mathrm{t})\right.$,

$$
\left.\mathrm{N}\left(\mathrm{Ax}_{2 \mathrm{n}-2}, \mathrm{Tz}, \mathrm{t}\right), \mathrm{N}\left(\mathrm{Ax}_{2 \mathrm{n}-2}, \mathrm{Bz}, \mathrm{t}\right), \mathrm{N}\left(\mathrm{S} \mathrm{x}_{2 \mathrm{n}-2}, \mathrm{Bz}, \mathrm{t}\right)\right\}
$$

Taking limit as $n \rightarrow \infty$, we get

$$
\begin{aligned}
\mathrm{N}(\mathrm{z}, \mathrm{Bz}, \mathrm{kt}) & \leq \max \{\mathrm{N}(\mathrm{z}, \mathrm{Tz}, \mathrm{t}), \mathrm{N}(\mathrm{z}, \mathrm{z}, \mathrm{t}), \mathrm{N}(\mathrm{Bz}, \mathrm{Tz}, \mathrm{t}), \mathrm{N}(\mathrm{z}, \mathrm{Tz}, \mathrm{t}), \mathrm{N}(\mathrm{z}, \mathrm{Bz}, \mathrm{t}), \mathrm{N}(\mathrm{z}, \mathrm{Bz}, \mathrm{t})\} \\
& =\max \{\mathrm{N}(\mathrm{z}, \mathrm{Bz}, \mathrm{t}), 1,1, \mathrm{M}(\mathrm{z}, \mathrm{Bz}, \mathrm{t}), \mathrm{N}(\mathrm{z}, \mathrm{Bz}, \mathrm{t}), \mathrm{N}(\mathrm{z}, \mathrm{Bz}, \mathrm{t})\},
\end{aligned}
$$

$\mathrm{N}(\mathrm{z}, \mathrm{Bz}, \mathrm{kt}) \leq \mathrm{N}(\mathrm{z}, \mathrm{Bz}, \mathrm{t})$.

Now, from (12), (13) and Lemma (2.14), we get $\mathrm{Bz}=\mathrm{z}$.

Therefore, we have $\mathrm{Tz}=\mathrm{Bz}=\mathrm{z}$.

Hence, we get $z$ is a common fixed point of B and T. From (9) and (14), we get

$\mathrm{Az}=\mathrm{Sz}=\mathrm{Bz}=\mathrm{Tz}=\mathrm{z}$. So $\mathrm{z}$ is a common fixed point of $\mathrm{A}, \mathrm{B}, \mathrm{S}$, and $\mathrm{T}$.

For uniqueness, Let $\mathrm{w}$ be the another common fixed point then $\mathrm{Aw}=\mathrm{Bw}=\mathrm{Sw}=\mathrm{Pw}=\mathrm{w}$

$M(A z, B w, k t) \geq \min \{M(S z, T w, t), M(A z, S z, t), M(B w, T w, t), M(A z, T w, t)$

$\mathrm{M}(\mathrm{Az}, \mathrm{Bw}, \mathrm{t}), \mathrm{M}(\mathrm{Sz}, \mathrm{Bw}, \mathrm{t})\}$

$$
=\min .\{\mathrm{M}(\mathrm{Az}, \mathrm{Bw}, \mathrm{t}), 1,1, \mathrm{M}(\mathrm{Az}, \mathrm{Aw}, \mathrm{t}), \mathrm{M}(\mathrm{Az}, \mathrm{Bw}, \mathrm{t}), \mathrm{M}(\mathrm{Az}, \mathrm{Bw}, \mathrm{t})\},
$$

$\mathrm{M}(\mathrm{Az}, \mathrm{Bw}, \mathrm{kt}) \geq \mathrm{M}(\mathrm{Az}, \mathrm{Bw}, \mathrm{t})$.

Also, we have

$\mathrm{N}(\mathrm{Az}, \mathrm{Bw}, \mathrm{kt}) \leq \max .\{\mathrm{N}(\mathrm{Sz}, \mathrm{Tw}, \mathrm{t}), \mathrm{N}(\mathrm{Az}, \mathrm{Sz}, \mathrm{t}), \mathrm{N}(\mathrm{Bw}, \mathrm{Tw}, \mathrm{t}), \mathrm{N}(\mathrm{Az}, \mathrm{Tw}, \mathrm{t})$, $\mathrm{N}(\mathrm{Az}, \mathrm{Bw}, \mathrm{t}), \mathrm{N}(\mathrm{Sz}, \mathrm{Bw}, \mathrm{t})\}$

$\mathrm{N}(\mathrm{Az}, \mathrm{Bw}, \mathrm{kt}) \leq \mathrm{N}(\mathrm{Az}, \mathrm{Bw}, \mathrm{t})$.

$$
=\max .\{\mathrm{N}(\mathrm{Az}, \mathrm{Bw}, \mathrm{t}), 0,0, \mathrm{~N}(\mathrm{Az}, \mathrm{Aw}, \mathrm{t}), \mathrm{N}(\mathrm{Az}, \mathrm{Bw}, \mathrm{t}), \mathrm{N}(\mathrm{Az}, \mathrm{Bw}, \mathrm{t})\} \text {, }
$$

From (15), (16) and Lemma (2.14), we get $A z=B w$, this implies $A z=A w$ and hence $z$ is a unique fixed point.

Example 3.2. Let $(X, M, N, *, \diamond)$ be a intuitionistic fuzzy metric, where $X=[2,20]$

$M(x, y, t)=\frac{t}{t+d(x, y)}, N(x, y, t)=\frac{d(x, y)}{t+d(x, y)}$ and $d$ is the Euclidean metric on $X$.

Define $\mathrm{A}, \mathrm{B}, \mathrm{S}$ and $\mathrm{T}: \mathrm{X} \rightarrow \mathrm{X}$ as follows;

$\mathrm{Ax}=2$ for all $\mathrm{x}$;

$\mathrm{Bx}=2$ if $\mathrm{x}<4$ and $\geq 5 \mathrm{Bx}=3+\mathrm{x}$ if $4 \leq \mathrm{x}<5$;

$\mathrm{Sx}=$ if $\mathrm{x} \leq 8, \mathrm{Sx}=8$ if $\mathrm{x}>8$;

$\mathrm{Tx}=2$ if $\mathrm{x}<4$ or $\geq 5, \mathrm{Tx}=5+\mathrm{x}$ if $4 \leq \mathrm{x}<5$.

Then A, B, S and T satisfy all the conditions of the above theorem and have a unique common fixed point $\mathrm{x}=2$.

Remarks: Our result is also true for the pair of mappings (A, S) is compatible of type (E) in place of compatible mapping of type $(\mathrm{K})$. Our result extends and generalizes the results of $\mathrm{M}$. Verma and 
R.S. Chandel [13], Jha et al.[11] Manandhar et al. and improve the result of Manandhar et al. [10]. Also, our result improves other similar results in literature.

\section{REFERENCES}

[1]. George, P. Veeramani, On some results in fuzzy metric spaces Fuzzy Sets and Systems, 64 (1994), 395-399.

[2]. Schweizer, Sklar, “A. Statistical metric spaces”, Pacific J. of Math., 10 (1960) 314-334.

[3]. Alaca, Turkoglu.D, Yildiz.C, "Fixed points in intuitionistic fuzzy metric Spaces", Chaos, Solitons and Fractals, 29(2006), 1073-1078.

[4]. G. Jungck and B. E. Rhoades, Fixed Point for Set Valued functions without Continuity, Indian J. Pure Appl. Math., 29(3), (1998), 771- 779.

[5]. G. Jungck, Compatible mappings and common fixed points, Internat. J. Math. Math. Sci., 9(1986), pp. 771-779.

[6]. G. Jungck, P. P. Murthy and Y. J. Cho, Compatible mappings of type (A) and common fixed points, Math. Japonica, 38(1993), 381-390.

[7]. H. K. Pathak, Y. J. Cho, S. S. Chang and S. M. Kang, Compatible mappings of type (P) and fixed point theorem in metric spaces and Probabilistic metric spaces, Novi Sad J. Math., Vol. 26(2)(1996), 87-109.

[8]. J.H. Park, "Intuitionistic fuzzy metric spaces", Chaos, Solitons \& Fractals 2(2004), 1039-1046.

[9]. K. B. Manandhar, K. Jha and G. Porru. Common Fixed Point Theorem of Compatible Mappings of Type (K) in Fuzzy Metric Space, Electronic J. Math. Analysis and Appl, 2(2)(2014), 248-253.

[10]. K. B. Manandhar, K. Jha and H. K. Pathak, A Common Fixed Point Theorem for mpatible Mappings of Type (E) in Fuzzy Metric space, Appl Math. Sci, 8 (2014), 2007 - 2014.

[11]. K. Jha, V. Popa and K.B. Manandhar, A common fixed point theorem for compatible mapping of type (K) in metric space, Internat. J. of Math. Sci. \& Engg. Appl. (IJMSEA), 8 (I) ( 2014), 383-391.

[12]. L.A. Zadeh, Fuzzy sets, Inform and Control 8 (1965), 338-353.

[13]. M. Verma and R. S. Chandel, Common fixed point theorem for four mappings in intuitionistic fuzzy metric space using absorbing Maps, IJRRAS 10 (2) (2012), 286 - 291.

[14]. O. Kramosil and J. Michalek, Fuzzy metric and statistical metric spaces, Kybernetika 11 (1975), 326-334.

[15]. R. P. Pant, A common fixed point theorem under a new condition, Indian. J. Pure Appl. Math., 30 (2) (1999) 147-152.

[16]. S. Sharma., Kutukcu,and R.S. Rathore, "Common fixed point for Multivalued mappings in intuitionistic fuzzy metricspace", Communication of Korean Mathematical Society, 22 (3),(2007), 391-399.

[17]. Y.J. Cho, H.K. Pathak, S.M. Kang, J.S. Jung, Common fixed points of compatible maps of type $(\beta)$ on fuzzy metric spaces, Fuzzy Sets and Systems 93 (1998), 99-111. 